\title{
Une Simulie nouvelle pour la Faune Française
}

\author{
Par Jean GIUDICELLI
}

En 1923, l'éminent diptérologiste anglais Edwards, étudiant des Simulies d'Algérie que lui avait envoyées Sergent, remarquait que les nymphes de Simulium equinum (Linné) étaient différentes des nymphes de cette même espèce récoltées en Angleterre par le fait que les six filaments centraux de l'appareil respiratoire étaient plus grêles. Il signalait en outre que le même type de nymphe existait éga!ement en Palestine et en Macédoine, ce qui l'amenait à penser qu'il s'agissait là d'une forme méditerranéenne de Simulium equinum.

En 1925, Puri, suivant l'idée d'Edwards, appelait cette forme Simulium equinum mediterraneum et il donnait une description complète de la nymphe où il apparaît que, outre le caractère distinctif signalé par Edwards, chacun des filaments grêles possède une constriction basale au niveau de laquelle la paroi du tube est annelée. En 1933, Puri trouvait cette Simulie dans le Nord-Ouest de l'Inde ; depuis Zivkovic (1952) l'a recueillie assez abondante en Yougoslavie, également Rivosecchi (1961) en Italie. Elle était inconnue jusqu'à présent de la faune française.

Au mois de mars 1962, Mlle Manier récoltait dans le cours d'eau la Mosson (affluent de l'Hérault), près de Montpellier, au lieu dit « Point du jour », des larves et des nymphes qu'elle m'envoyait. Je relevais dans cette faune: Simulium aureum Fries, $S$. bezzii Corti, $S$. ornatum nitidifrons Edwards, enfin des larves et des nymphes de $S$. equinum mediterraneum.

Grenier (communication verba'e) a déterminé cette Simulie dans un lot de larves et de nymphes rapportées en 1960 de la rivière le Coulon (affluent de la Durance), près de Cavaillon (Vaucluse), par Rageau et Le Berre. Cette récoite a été mentionnée dans un rapport non publié des stagiaires entomo'ogistes de l'O.R.S.T.O.M. Ce sont là les deux premières stations pour cette Simulie en France.

Dans le matériel de la Mosson, j'ai isolé les larves correspondant aux nymphes de S. equinum mediterraneum. Ces larves, comme toutes celles des autres espèces du groupe equinum (que certains auteurs désignent sous le nom générique de Wilhelmia Enderlein), possèdent des épines tégumentaires formées de deux à quatre branches ; les autres caractères morphologiques correspondent à ceux que l'on trouve dans les descriptions de la larve de cette variété. Cependant les larves de la Mosson possèdent sur chacun de leurs cinq premièrs segments abdominaux une paire de tubercules dorsaux très nets ainsi qu'on peut le voir sur la photo. Ce caractère morphologique est surprenant car il n'existe 


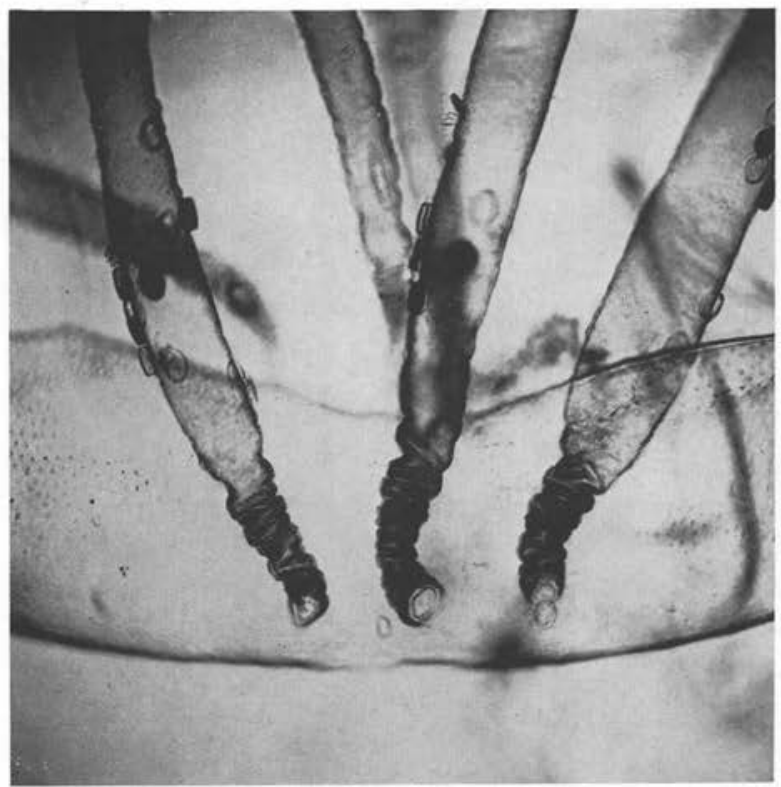

Simulium equinum mediterraneum. Localité : la Mosson (Hérault) Fig. 1. - Cliché 1. Base des filaments centraux de l'appareil respiratoire

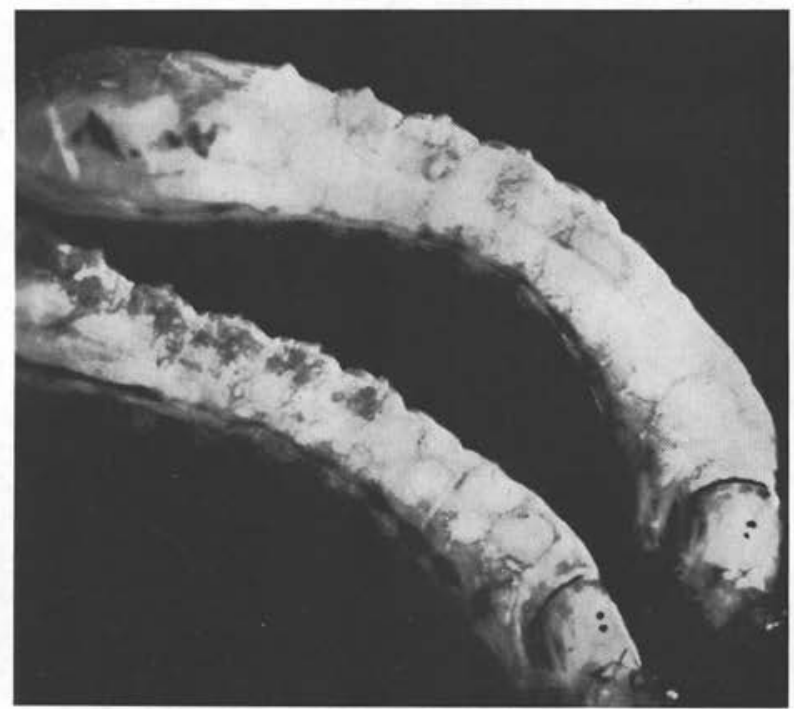

FIG. 2. - Cliché 2. Larves avec leurs tubercules tégumentaires dorsaux 
jusqu'alors, d'après Grenier et Féraud (1960), que trois espèces de Simulies dans le monde dont les larves présentent de tels tubercules: ce sont celles de $S$. damnosum Théobald (Afrique), S. varicorne Edwards (Sumatra) et de $S$. maculatum Meigen (région du Danube).

Je me suis assuré par ailleurs que les nymphes et les génitalia ex nympha chez la Simulie de la Mosson sont en tous points identiques à ceux décrits pour la variété typique. J'ai pensé alors que la présence de ces tubercules dorsaux était un caractère des larves de $S$. equinum mediterraneum, caractère qui n'avait pas retenu l'attention précédemment. J'ai posé la question à Zivkovic et à Grenier pour savoir si les larves de cette Simulie, qu'ils avaient eu l'occasion d'observer, présentaient ce caractère ; leur réponse fut négative.

Je considère actuellement que cette popu'ation de S. equinum mediterraneum représente une forme un peu différente de la variété type. Je ne pense pas qu'il faille lui donner une appellation propre, mais cette particularité des larves est à souligner et il serait intéressant d'en rechercher l'extension géographique.

\section{Bibliographie}

Edwards (F. W.), 1923. - On some algerian species of Simulium. Arch. Inst. Pasteur, Algérie, $1, \mathrm{n}^{\circ} 4,647-653$.

Grenier (P.) et Féraud (L.), 1960. - Les larves à tubercules dorsaux chez les Simulies (S. damnosum Théo.; S. (?) varicorne, Edw.; S. maculatum Meig.). Bull. Soc. Pathol. exotique, 53, $\mathrm{n}^{\circ}$ 2, 332-338.

PURI (J.-M.), 1925. - A note on the early stages of a species of Simulium from Tunis, S. equinum var. mediterraneum, nov. (Diptera, Simuliidae). Ann. Mag. Nat. Hist., 16, ser. 9, 253-255.

-, 1933. - Studies on Indan Simuliidae. Part IX. Ind. Journ. med. Res., 21, $\mathrm{n}^{\circ}$ 11-16.

Rrvosecchi (L.), 1961. - Contributo alla conoscenza dei Simulidi italiani. I. Reperti in provincia di Latina. Rivista di Parassitol., 22, $\mathrm{n}^{\circ}$ 1, 27-54.

Zivkovic (V.), 1952. - Contribution à l'étude des Simuliides de Yougoslavie. C.R. Acad. Sc. Serbie, Sect. Med., 5, 9-36.

(Laboratoire de Zoologie Générale, Faculté des Sciences de Marseille) 